\title{
A Truncated Form of the Polarity Protein SCRIB Induces Apoptosis in MDA-MB231 Cells by Regulating Components of Several Signalling Pathways
}

\author{
Maria Makkou ${ }^{1,2}$, Takis Makatounakis ${ }^{2}$ and Joseph Papamathekis ${ }^{1,2 *}$ \\ ${ }^{1}$ Department of Biology, University of Crete, Greece \\ ${ }^{2}$ Institute of Molecular Biology and Biotechnology, Foundation for Research and Technology-Hellas (FORTH), Greece
}

Submission: : July 15, 2019; Published: July 26, 2019

"Correspondence Author: J Papamatheakis, Department of Biology, University of Crete, N Plastira 100, 70013 Heraklion, Crete, Greece

\begin{abstract}
Polarity proteins are fundamental to the establishment and maintenance of breast tissue integrity, whereas alterations of their amino acid sequence and protein structure can drive breast cancer formation and progression. Scrib is a multi-functional scaffold protein whose proper localization within the cell is pivotal for its correct function. We report here that the ectopic expression of a truncated form of the polarity protein Scrib, mainly composed of the LRR domain, drives MDA-MB231 breast cancer cells to caspase 3-mediated apoptotic cell death. Furthermore, we identify the truncated Scrib as a regulator of JNK/c-Jun, Notch and Wnt/ $\beta$-catenin signalling pathways. Our results indicate that the ectopic expression of the truncated Scrib in a breast cancer cell line with a specific background of genetic mutations (KRAS, AFRGAP1, BRAF, MYCL) activates a program of intracellular molecular alterations and promotes apoptosis.
\end{abstract}

Keywords: Cell polarity; Scrib; Breast cancer; Apoptosis

\section{Introduction}

Cell polarity is essential for the structure and function of all body tissues. Cell polarity determines crucial cellular functions such as the establishment of the apico-basal cell polarity, asymmetric cell division and cell migration. Epithelial cells are a typical example of polarized cells (apico-basal polarity) with the apical surface exposed to the lumen and the basal surface attached to the basement membrane. Three main protein complexes are responsible for the establishment of cell polarity, the Crumbs (Crumbs3-PALS1-PATJ) and Par (Par3-Par6-aPKC) complexes identify the apical domain of the cell, while the Scribble complex [lethal giant larvae (Lgl) - Scribble (Scrib) Disc large (Dlg)] defines the basal domain Wen \& Zhang [1].

The polarity protein Scrib is known to interact with structural and signalling proteins, regulating cell-cell adhesion, cytoskeleton remodeling and the activation state of several signalling pathways Feigin \& Muthuswamy [2], McCaffrey LM \& Macara [3]. Scrib consists of an N-terminal region with 16-leucine-rich repeats (LRR) that is responsible for its membrane targeting, following $4 \mathrm{PDZ}$ domains that interact with proteins such as ZO-2, APC, $\beta$-PIX, zyxin family and a C-terminal region that contains three spectrin binding motifs Stephens et al. [4]. Alterations in the regulation or sequence of scrib gene leads to abnormal protein levels and subcellular localization Lin et al. [5], Wan et al. [6]. Various point mutations or gene amplifications have been reported in several cases of cancer, including breast cancer (https://www.cbioportal.org/, Lin et al. [5].

Scrib mutations that lead to truncated Scrib versions missing the LRR domain result in a phenotype that mimics Scrib loss Zeitler et al. [7]. In Drosophila such mutations lead to complete deregulation of cell polarity and growth control, highlighting the key role of the LRR domain in Scrib-mediated functions Zeitler et al. [7]. In comparative terms, Drosophila mutants missing the PDZ domains exhibit a phenotype similar to low level expression if Scrib Zeitler et al. [7]. According to genomic data from the Cancer Genome Atlas (TCGA), several human cancers (including breast cancer) harbor nonsense mutations in scrib, leading to the expression of truncated forms of the protein. Several studies support the involvement of Scrib in human cancers. Loss of Scrib in cooperation with myc oncogene has been correlated with blocking apoptosis, cell transformation and breast cancer 
formation Zhan et al. [8]. Furthermore, mutations that lead to loss of Scrib act complementarily with mutations leading to Ras and Notch activation, which promote tumorigenesis and tumor progression Brumby et al. [9]. Importantly, although Scrib is known to interfere with various signaling pathways to control crucial cellular functions and to promote tumorigenesis, whether truncated versions of Scrib play a role in similar processes is not known. In this study, we use a triple-negative breast cancer cell model to investigate how overexpression of a truncated version of Scrib protein affects components of signaling pathways that are involved in apoptosis. Our data suggest that the truncated Scrib protein encompassing amino-acids $94-494$ is a regulator of multiple signalling events and indicate a key role in apoptotic cell death in this breast cancer model.

\section{Materials and Methods}

\section{Cell culture and Treatments}

The MDA-MB231 cell line was purchased from ATCC. Cells were cultured in high glucose Dulbecco's modified Eagle's medium (DMEM) (Life Technologies \#41965-062) supplemented with 10\% heat-inactivated fetal bovine serum (FBS) (PAA Gold, GE Healthcare \#A15151) and 1\% penicillin-streptomycin (Life Technologies \#15140122). Cells were cultured in a humified incubator at $37{ }^{\circ} \mathrm{C}$ under $5 \% \mathrm{CO}$. To induce expression of Scrib $94-494$, cells were treated with $1 \mu \mathrm{g} / \mathrm{ml}$ doxycycline (dissolved in distilled water). For the generation of doxycycline (dox) inducible cell lines, the pLenti CMV rtTA3 Blast (w756-1) plasmid (Addgene \#26429) was introduced into MDA-MB 231 cells and transfected cells were selected using $5 \mu \mathrm{g} / \mathrm{ml}$ blasticidin (Sigma Aldrich). Subsequently, a dox inducible Scrib94-494RFP expression vector was introduced into MDA-MB231 cells followed by puromycin $(5 \mu \mathrm{g} / \mathrm{ml})$ (Sigma Aldrich) selection.

\section{Plasmid Constructs}

For the Scrib 94-494 RFP tagged expression vector, Scrib 94494 was obtained from the pLK01 plasmid (Addgene, \#37252) by SalI/StuI restriction enzyme digestion and subcloned into the SmaI/SalI sites of the JRed-C2 vector (Addgene, \#54788). Finally, the JRed-C2_Scrib 94-494 plasmid was digested with BamHI and NheI restriction enzymes and the Scrib-RFP fragment was subcloned into XbaI and BamHI sites of the pMA2780 vector (Addgene, \#25438). The integrity of the final construct was confirmed by sequencing.

\section{Antibodies}

Antibodies against cleaved caspase-3 (\#9661S), phosphoc-Jun (\#3270), Notch1 (\#3608), Notch2 (\#5732) and Notch3 (\#5276) were purchased from Cell Signaling Technology. Antibodies against Scrib (C-20) (sc-11049), phospho-JNK (G-7) (sc-6254), JNK (D-2) (sc-7345) and $\beta$-catenin (E-5) (sc-7963) were purchased from Santa Cruz. The anti-Killer Red (AB961) and $\alpha$-tubulin (\#T9026) antibodies were purchased from
Evrogen and Sigma, respectively. All antibodies were used in 1:1000 dilution for western blotting.

\section{Virus Production}

For lentivirus production, the lentiviral constructs and second-generation packaging plasmids pMD2.G (Addgene, \#12259) and psPAX2 (Addgene, \#12260) were co-transfected into HEK293T cells. Lentivirus-containing supernatant was collected 48 and $72 \mathrm{~h}$ after transfection and filtered through a $0.45 \mathrm{~mm}$ filter before use. Transfections were performed using the calcium phosphate DNA precipitation method Chen [10].

\section{Western Blot}

Whole cell protein extracts were prepared by lysing the cells in-well with ice-cold RIPA lysis buffer $(50 \mathrm{mM}$ Tris- $\mathrm{HCl}$ $\mathrm{pH} 8.0,150 \mathrm{mM} \mathrm{NaCl}, 2 \mathrm{mM} \mathrm{MgCl} 2,2 \mathrm{mM} \mathrm{CaCl} 2,0,5 \%$ sodium deoxycholate, 1\% NP40, 0,1\% SDS, 10\% Glycerol, 1x PhosSTOP phosphatase inhibitors and $1 \mathrm{x}$ Complete protease inhibitors), for $10 \mathrm{~min}$ on ice and then for $20 \mathrm{~min}$ on a rotating wheel at $40 \mathrm{C}$. The lysates were clarified by centrifugation (10 min, $16000 \mathrm{rpm}$, 40C) and total protein concentration was estimated using a Bradford reagent according to the manufacturer's instructions. The supernatant was transferred to a new tube and SDS loading buffer was added before boiling for $10 \mathrm{~min}$ at $95 \mathrm{oC}$. $30 \mu \mathrm{g}$ of protein were separated on SDS-PAGE gels and transferred to

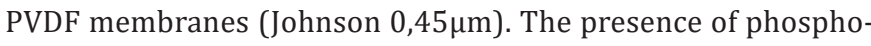
and total proteins was detected using the appropriate antibodies. Blots were developed with the ECL system (Thermo Scientific).

\section{Cell Cycle Analysis}

For cell cycle analysis, 100.000 cells from each sample were trypsinized, washed with PBS, treated with $200 \mu \mathrm{g} / \mathrm{ml}$ RNAse A (Qiagen) for $30 \mathrm{~min}$ at $370 \mathrm{C}$ and stained with propidium iodide (PI -Sigma) according to the manufacturer's instructions. The analysis was conducted by flow cytometry (FACS Calibur analyzer). The cell cycle profile was further analyzed using the ModFit LT software.

\section{Immunofluorescence}

The cells were fixed with $4 \%$ paraformaldehyde for $15 \mathrm{~min}$ at room temperature. The cells were washed 3 times with $0.05 \%$ Tween-PBS and permeabilized in 0.5\% NP40-PBS (Roche) for 5 min at room temperature followed by three rinses. Thereafter, samples were blocked with PBS 1\% BSA for $1 \mathrm{~h}$. Then, the samples were incubated with primary antibodies diluted in PBS with $3 \%$ bovine serum albumin (BSA, Sigma) for $1 \mathrm{~h}$ at room temperature. After washing 3 times with PBS, the samples were incubated with fluorophore-conjugated secondary antibodies diluted in PBS with 3\% BSA at room temperature for $1 \mathrm{~h}$. Then the cells were washed for another 3 times. Finally, cells were mounted with ProLong Diamond Antifade Mountant with DAPI (ThermoFisher, \#P36962). Confocal microscopy was carried out on a Laser-scanning confocal Leica SP8-X. 
Results

\section{Overexpression of the truncated SCRIB 94-494 induces morphological changes in MDA-MB231 cells}

Dysregulation of oncogenic signaling pathways, such as Ras, has been indicated to correlate with Scrib protein mutations, leading to tumor formation and progression Dow et al. [11]. Data from the Cancer Genome Atlas (TCGA) database indicate that several cancers including breast cancer, harbor mutations in the sequence of scrib that lead to truncated forms of the protein (Figure 1). For instance, mutations that result to premature stop codons and lead to scrib alleles that encode the N-terminal LRR domain and lack the C-terminal part of the protein are found in breast invasive ductal carcinoma, in melanoma, as well as in uterine endometroid carcinoma (Figure 1b) (https://www. cbioportal.org/). Furthermore, Metodieva et al. [12] showed that some breast cancers apply distinct scrib exon usage pattern, which results in overexpression of conserved exons encoding the N-terminal LRR domain and loss of the exons encoding the
C-terminus, therefore lacking the cell cycle checkpoint function of the C-terminal part of Scrib.

To examine the role of a truncated scrib mutant mainly consisting of the LRR domain of Scrib (Figure 1a), we used MDAMB231, a highly aggressive triple negative human breast cancerderived cell line. In these cells which scrib gene is amplified in one chromosome while it is deleted in its chromosomal pair. We employed a tetracycline inducible system to control the ectopic expression of Scrib94-494 - RFP in these cells. Following dox treatment, Scrib 94-494 over-expressing cells demonstrated a rounder morphology and a decrease in cellcell contacts as compared to un-induced controls (Figure 1c). Immunofluorescence analysis using a Scrib polyclonal antibody, which recognizes the C-terminal part of the protein, revealed that endogenous Scrib is localized both at the cell surface and the cytoplasm of the MDA-MB231 Scrib 94-494 un-induced cells. Upon dox treatment we observed diffuse cytoplasmic localization of endogenous Scrib which colocalized with the ectopically expressed truncated Scrib (Figure 1c).

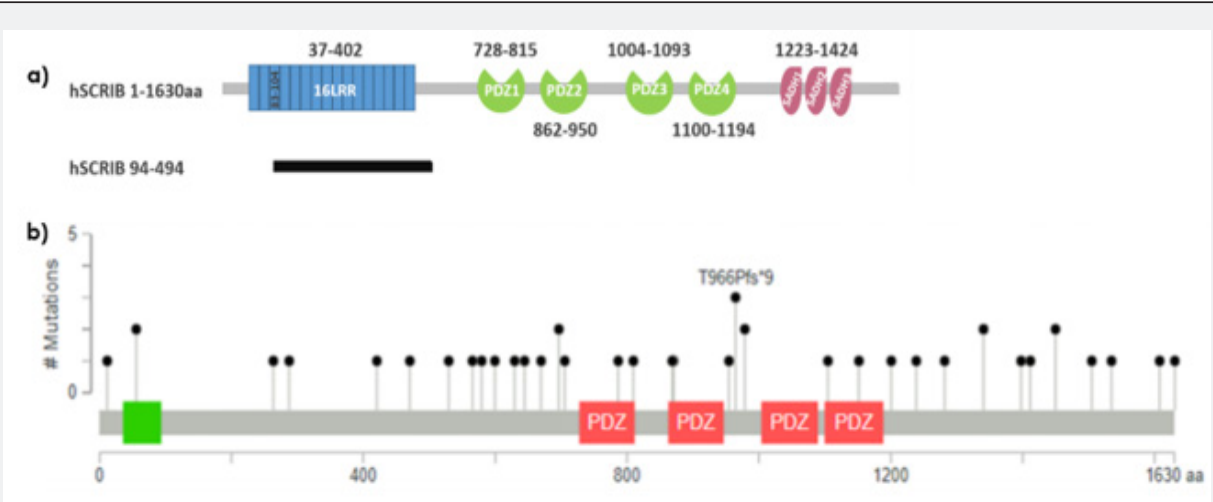

c)
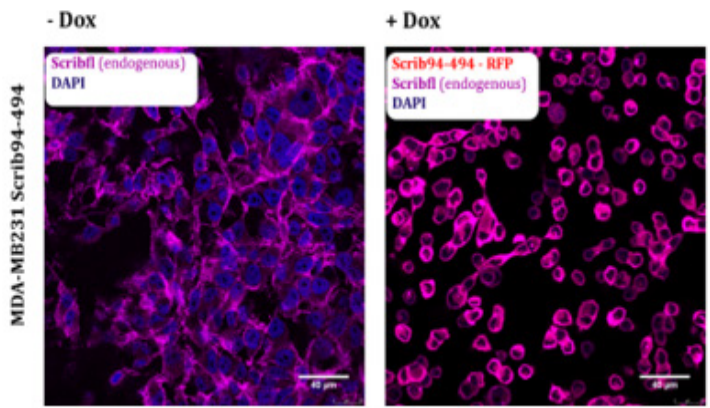

Figure 1:

a) Schematic of the SCRIB domains and the truncated SCRIB $94-494$ construct used in this study.

b) Truncating mutations in SCRIB, in human cancer.

c) Representative images illustrating the endogenous SCRIB and the ectopically expressed Scrib 94-494 in MDA-MB231 cells.

\section{Overexpression of the truncated SCRIB 94-494 drives MDA-MB231 to Cell Cycle Arrest and Apoptosis}

We observed that the induction of Scrib 94-494 was accompanied by reduction in the total cell number. To examine if this reduction was associated with cell cycle arrest, the Scrib 94494 expressing cells and the un-induced controls were stained with PI and analyzed by flow cytometry. We found that Scrib 94494 expressing cells underwent a G0/G1 cell cycle arrest (Figure 2a). The role of Scrib 94-494 in apoptosis was investigated by Western blotting analysis of cleaved caspase-3. Analysis of whole cell lysates indicated the presence of cleaved caspase- 3 in Scrib 94-494 expressing cells, suggesting the involvement of the 


\section{Cancer Therapy \& Oncology International Journal}

truncated Scrib in caspase-mediated apoptosis (Figure 2b). Our combined results indicate that the LRR domain of Scrib might

have an important role in cell cycle arrest as well as cell death induction.

a)

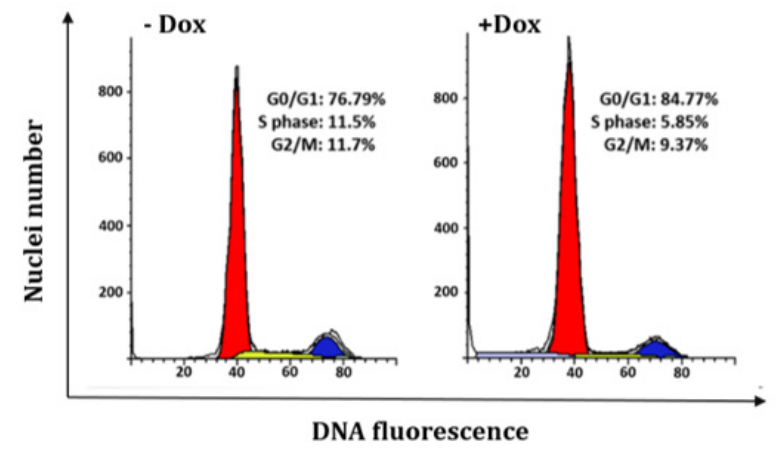

b)

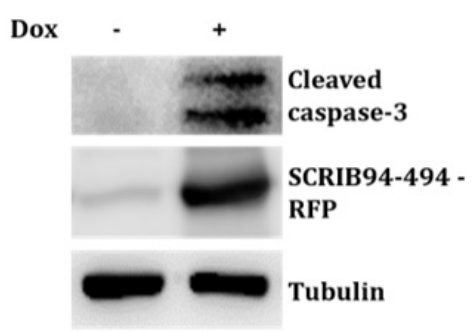

Figure 2: SCRIB 94-494 -mediated cell growth arrest and apoptosis in human breast cancer cell lines. a) Cell cycle analysis by flow cytometry Cell cycle distribution of propidium iodide (PI)-labeled cells was analyzed by flow cytometric analyses after $72 \mathrm{~h}$ of SCRIB $94-$ 494 induction by doxycycline. Western blot analysis showing SCRIB 94-494-tagged RFP ectopic expression in an inducible MDA-MB231 system, and activation of caspase-3.

Ectopic expression of the truncated SCRIB 94-494 influences JNK/c-Jun, Notch and Wnt/ $\beta$-catenin signalling pathways

It is well established that $s c r i b$ is a key regulator of several signal transduction pathways Stephens et al. [4]. It was previously shown in Drosophila that JNK normally promotes apoptosis in scrib mutant cells, whereas scrib mutants lead to tumour overgrowth and invasion by acting synergistically with the activated Ras or Notch signaling pathways Brumby et al. [9] \& Leong et al. [13]. To examine the role of Scrib 94-494 expression in JNK (c-Jun N-terminal kinase)/c-Jun and Notch signaling pathways, we carried out western blot analysis of whole-cell lysates of Scrib 94-494 expressing cells. Western blot analysis revealed activation of the (JNK)/c-Jun signaling axis in Scrib 94-494 expressing cells, as indicated by phosphorylation status of JNK and c-Jun signaling proteins (Figure 3). To investigate the impact of ectopic expression of Scrib 94-494 on Notch signaling, we used Notch isoform-specific antibodies that detect both full-length and the NTM region of Notch, consisting of a short extracellular juxtamembrane peptide, a transmembrane sequence and the intracellular domain (NICD). Our results indicated that the protein levels of both full-length and the NTM region of Notch1, Notch2 as well as Notch3 were reduced in Scrib 94-494 expressing cells (Figure 3). Overall, these data suggest that Scrib 94-494 activates JNK/c-Jun signalling axis by post-translational phosphorylation of its components, whereas influences Notch signalling regulating Notch protein levels.
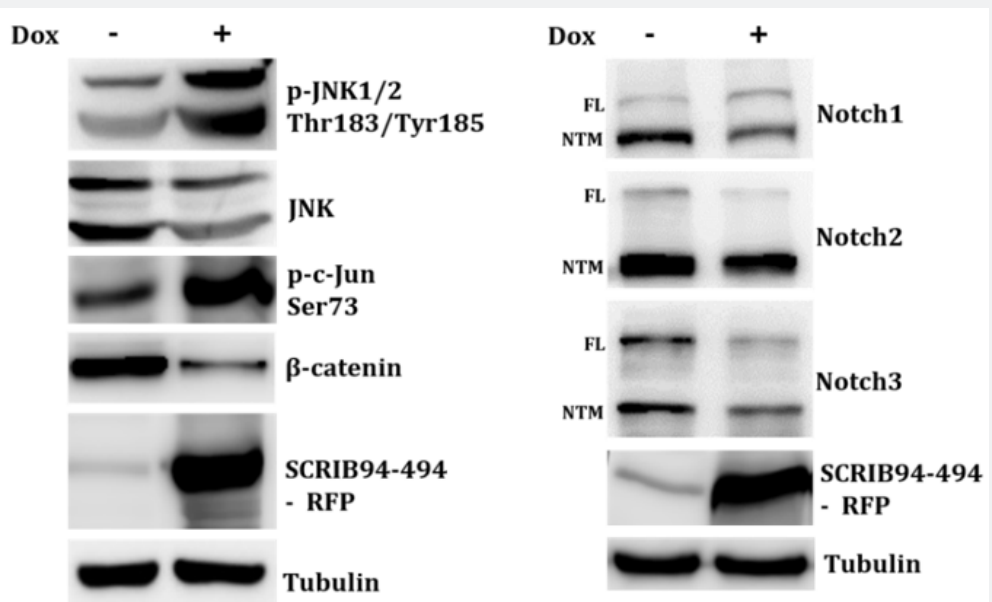

Figure 3: Western blot analysis of whole-cell lysates of MDA-MB231 in which SCRIB 94-494 is induced in the presence of doxycycline (+Dox). When doxycycline is present:

i) The JNK/c-Jun signaling axis is potently activated.

ii) Total protein levels of $\beta$-catenin are reduced.

iii) Notch protein levels are reduced. 


\section{Cancer Therapy \& Oncology International Journal}

Several studies support the crosstalk between MAPK signalling and the canonical Wnt signalling pathway Bikavilli et al. [14]. In the absence of the Wnt ligand, $\beta$-catenin phosphorylation results in its proteasomal degradation MacDonald et al. [15]. To assess $\beta$-catenin protein levels in Scrib 94-494 expressing cells, we carried out western blot analysis of whole cell lysates. Significant reduction of $\beta$-catenin protein levels was observed in Scrib 94-494 expressing cells that could be driven by the $\beta$-catenin destabilization. Collectively, our data indicate that a Scrib 94-494-mediated molecular mechanism positively regulates the JNK/c-Jun signalling axis, while at the same time reduces Notch and $\beta$-catenin total protein levels in MDA-MB231 cells [16-20].

\section{Discussion}

Our findings suggest that a truncated form of Scrib possesses a key regulatory role in signal transduction by affecting components of the JNK/c-Jun, Notch and Wnt/ $\beta$-catenin signaling pathways in a breast cancer cell model that harbor multiple oncogenic mutations. It was previously shown that JNK activation normally promotes apoptosis of scrib mutant cells in Drosophila, whereas scrib mutations can lead to tumour overgrowth and invasion in cells with constitutively activated Ras or Notch signaling Brumby et al. [9] \& Leong et al. [13]. Furthermore, Scrib activates JNK/c-Jun signalling pathway and promotes apoptosis in a normal mammary epithelia cell line Zhan et al. [8]. In our in vitro model, we have shown that a truncated scrib mutant mainly composed of the LRR domain, which is crucial for its proper localization, activates JNK/c-Jun signalling pathway while reduces Notch and $\beta$-catenin protein levels. Additionally, the truncated Scrib induces cell cycle arrest at G1/G0 phase and promotes apoptosis in MDA-MB231 human breast cancer cells. Based on our findings we speculate that overexpression of Scrib 94-494 might drive apoptosis of MDAMB231 cells via regulation of components of several signalling pathways.

\section{Conclusion}

In conclusion, our results demonstrate that a truncated form of Scrib protein, mainly consisting of the LRR domain, is able to regulate JNK/c-Jun, Notch and Wnt/ $\beta$-catenin signalling pathways, providing hints for a crosstalk among them that leads cells to apoptotic cell death. The detection of truncated forms of scrib in human breast cancer specimen could find application as a prognostic marker of the disease and could provide a mean to assess easily the activation status of JNK, Notch and Wnt/ $\beta$ catenin signalling pathways; something that could be of utmost importance in clinical practice.

\section{Acknowledgement}

We would like to thank G. Vretzos for technical assistance.

\section{References}

1. Wen W, Zhang M (2017) Protein Complex Assemblies in Epithelial Cell Polarity and Asymmetric Cell Division. J Mol Biol 430(19): 3504-3520.
2. Feigin ME, Muthuswamy SK (2009) Polarity proteins regulate mammalian cell-cell junctions and cancer pathogenesis. Curr Opin Cell Biol 21(5): 694-700.

3. McCaffrey LM, Macara IG (2012) Signaling pathways in cell polarity. Cold Spring Harbor perspectives in biology 4(6): a009654.

4. Stephens R, Lim K, Portela M, Kvansakul M, Humbert PO (2018) The Scribble Cell Polarity Module in the Regulation of Cell Signaling in Tissue Development and Tumorigenesis J Mol Biol 430(19): 35853612 .

5. Lin WH, Asmann YW, Anastasiadis PZ (2015) Expression of polarity genes in human cancer. Cancer informatics 14: 15-28.

6. Wan S, Meyer AS, Weiler SME, Rupp C, Tóth M (2018) Cytoplasmic localization of the cell polarity factor scribble supports liver tumor formation and tumor cell invasiveness. Hepatology 67(5): 1842-1856.

7. Zeitler J, Hsu CP, Dionne H, Bilder D (2004) Domains controlling cell polarity and proliferation in the Drosophila tumor suppressor Scribble. J Cell Biol 167(6): 1137-1146.

8. Zhan L, Rosenberg A, Bergami KC, Yu M, Xuan Z, et al. (2008) Deregulation of scribble promotes mammary tumorigenesis and reveals a role for cell polarity in carcinoma. Cell 135(5): 865-878.

9. Brumby AM, Richardson HE (2003) scribble mutants cooperate with oncogenic Ras or Notch to cause neoplastic overgrowth in Drosophila. EMBO J 22(21): 5769-5779.

10. Chen Y (2012) Calcium Phosphate Transfection of Eukaryotic Cells. Bio 101: e86.

11. Dow LE, Elsum IA, King CL, Kinross KM, Richardson HE (2008) Loss of human Scribble cooperates with H-Ras to promote cell invasion through deregulation of MAPK signaling. Oncogene 27(46): 59886001.

12. Metodieva G, Adoki S, Lausen B, Metodiev MV (2016) Decreased Usage of Specific Scrib Exons Defines a More Malignant Phenotype of Breast Cancer with Worsened Survival. EBioMedicine 8: 150-158.

13. Leong GR, Goulding KR, Amin N, Richardson HE, Brumby AM (2009) Scribble mutants promote aPKC and JNK-dependent epithelial neoplasia independently of Crumbs. BMC Biol 7007-7-62.

14. Bikkavilli RK, Malbon CC (2009) Mitogen-activated protein kinases and Wnt/beta-catenin signaling: Molecular conversations among signaling pathways. Commun Integr Biol 2(1): 46-49.

15. MacDonald BT, Tamai K, He X (2009) Wnt/ $\beta$-Catenin Signaling: Components, Mechanisms, and Diseases. Dev Cell 17(1): 9-26.

16. Feigin ME, Akshinthala SD, Araki K, Rosenberg AZ, Muthuswamy LB (2014) Mislocalization of the cell polarity protein scribble promotes mammary tumorigenesis and is associated with basal breast cancer. Cancer research 74(11): 3180-3194.

17. Frank SR, Bell JH, Frödin M, Hansen SH (2012) A ßPIX-PAK2 complex confers protection against Scrib-dependent and cadherin-mediated apoptosis. Current biology 22: 1747-1754.

18. Hayward P, Kalmar T, Arias AM (2008) Wnt/Notch signalling and information processing during development. Development 135(3): 411-424.

19. Lien GS, Wu MS, Bien MY, Chen CH, Lin CH (2014) Epidermal growth factor stimulates nuclear factor-kappaB activation and heme oxygenase-1 expression via c-Src, NADPH oxidase, PI3K, and Akt in human colon cancer cells. PLoS One 9(8): e104891.

20. Young LC, Hartig N, Muñoz-Alegre M, Oses-Prieto JA, Durdu S (2013) An MRAS, SHOC2, and Scrib complex coordinates ERK pathway activation with polarity and tumorigenic growth. Mol Cell 52(5): 679-692. 


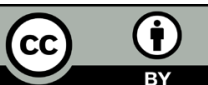

his work is licensed under Creative Commons Attribution 4.0 License DOI: 10.19080/CTOIJ.2019.14.555894

\section{Your next submission with Juniper Publishers} will reach you the below assets

- Quality Editorial service

- Swift Peer Review

- Reprints availability

- E-prints Service

- Manuscript Podcast for convenient understanding

- Global attainment for your research

- Manuscript accessibility in different formats ( Pdf, E-pub, Full Text, Audio)

- Unceasing customer service

Track the below URL for one-step submission https://juniperpublishers.com/online-submission.php 